\title{
Características morfométricas, rendimento e composição do filé de tilápia do Nilo, Oreochromis niloticus, da linhagem tailandesa, local e do cruzamento de ambas
}

\section{Morphometrics, fillet yield and fillet composition in Nile tilapia, Oreochromis niloticus, strains thai chitralada, Brazil local and their hybrid}

\author{
Julio Hermann Leonhardt ${ }^{*}$; Mauro Caetano Filho'; \\ Heitor Frossard²; Aleksey Machado Moreno ${ }^{3}$
}

\begin{abstract}
Resumo
As características morfométricas, rendimento e a composição do filé foram pesquisadas em tilápia do Nilo Oreochromis niloticus, das linhagens tailandesa chitralada (Tai), local (Local, Norte do Paraná, Brasil), e da proveniente do cruzamento de ambas ( $\mathrm{Hbr}$, macho tailandesa $\mathrm{x}$ fêmea local). Ao início do experimento os peixes (n: 900) apresentavam peso de $0,39 \pm 0,20 ; 0,41 \pm 0,22$ e $0,45 \pm 0,15 \mathrm{~g}$ e ao final 650,$67 ; 534,25$ e 360,00 g para as variedades Tai, Local e Hbr, respectivamente. Foram estabelecidas quatro razões morfométricas, sendo que a razão entre a altura da cabeça/ comprimento da cabeça da variedade Tai foi maior $(P<0,05)$ quando comparada às duas outras linhagens. A linhagem $\mathrm{Hbr}$ apresentou maior rendimento em filé $(39,05 \%)(P<0,05)$ quando comparada à Local $(38,00 \%)$ e Tai $(36,51 \%)$, que não diferiram entre si $(P>0,05)$. A composição centesimal do filé da linhagem Local apresentou menor teor de lipídeos $(1,88 \%)(P<0,05)$ quando comparada à $\mathrm{Hbr}(2,44 \%)$ e Tai $(2,96 \%)$, que também diferiram entre si, com menor teor de lipídeos para a $\operatorname{Hbr}(P<0,05)$. O teor em proteína bruta, cinzas e umidade do filé não apresentaram diferenças significativas $(P>0,05)$ entre as variedades.

Palavras-chave: Tilápia do Nilo, produção de filé, composição de filé, características morfométricas
\end{abstract}

\begin{abstract}
Morphometrics, fillet yield and fillet composition differences were researched in Nile tilapia, Oreochromis niloticus, strain thai-chitralada (Tai), Brazil (Local) Northern Paraná and their hybrid (Hbr, male Thailand $\mathrm{x}$ Brazilians female). The experiment was designed entirely randomly with three treatments (strains) and three repetitions per treatment in hapa nets in ponds. The initial weights were $0.39 \pm 0.20,0.45 \pm 0.22$ and $0.41 \pm 0.15 \mathrm{~g}$ for the strains Tai, Bras and Hbr, respectively. At the end of the experiment, the weights were $650.67,534.25$ and $360.00 \mathrm{~g}$ for the same previous sequence, with statistically significant differences between groups $(P<0.05)$. Four morphometric proportions were established to characterise each group.
\end{abstract}

\footnotetext{
1 Professor da Disciplina de Aquicultura e Piscicultura do Departamento de Ciências Fisiológicas, Centro de Ciências Biológicas, Universidade Estadual de Londrina, Campus Universitário, CP 6001, CEP 86051-970, Londrina- PR. Doutor em Aquicultura pela UNESP, Jaboticabal, SP; Coordenador e colaborador de projetos de pesquisa em Aquicultura; Co-autor do capítulo "Piscicultura” do Livro “A Bacia do Rio Tibagi. E-mail: leonhard@uel.br.

2 Estação de Piscicultura do Departamento de Biologia Animal e Vegetal, CCB, UEL.

3 Graduado em Medicina Veterinária pela UEL.

* Autor para correspondência.
} 
For the ratio (head height)/(head length), the Tai strain was largest $(P<0.05)$ as compared to the two other strains. The other morphometric proportions were not different $(P>0.05)$. The strain Hbr produced $(P<0.05)$ greater fillet yield $(39.05 \%)$ when compared to Local $(38.00 \%)$ and Tai $(36.51 \%)$, the latter two were not different from each other $(P>0.05)$. Considering fillet composition, Local strain had the least crude lipid content of $(1.88 \%)$, as compared to $\mathrm{Hbr}(2.44 \%)$ and Tai $(2.96 \%)$ which were significantly different $(P<0.05)$. Crude protein, ash and moisture of the fillets were not different $(\mathrm{P}>0.05)$.

Key words: Oreochromis niloticus, Nile tilapia, fillet yield, fillet composition, morphometric reasons

\section{Introdução}

As pesquisas genéticas e zootécnicas com tilápia historicamente têm sido direcionadas para: a) avaliação de espécies e linhagens em diferentes sistemas de cultivo, b) melhoria de performance em crescimento e ganho em peso, c) reversão sexual e produção de populações 100\% machos (POPMA; GREEN, 1990; MAIR et al., 1995; POPMA; LOVSHIN, 1996; TUAN; LITTLE; MAIR, 1998; ABUCAY et al., 1999; SURESH, 1999; DAN; LITTLE, 2000). Porém, estudos que visem ganho no rendimento do filé são escassos (RUTTEN; BOVENHUIS; KOMEM, 2004). Estes autores pesquisaram o rendimento de filé de três linhagens (linhagem chitralada, linhagem IDRC "Projeto do Asian Institute of Tecnology" e linhagem GIFT "Genetic improvement of farmed tilapias") de Oreochromis niloticus e encontraram diferenças significativas $(P<0,05)$ entre as mesmas com valores de $34,5 \%, 35,2 \%$ e $37,8 \%$ respectivamente.

Beniga e Circa (1997) estudaram o crescimento de três linhagens puras e uma linhagem local de $O$. niloticus em tanques-rede nas Filipinas e observaram que as linhagens puras apresentaram melhor taxa de crescimento que a local. Entretanto, a sobrevivência das linhagens puras foi inferior à local. Na Tailândia, Tuan, Little e Mair (1998) demonstraram que linhagens tailandesas e seus híbridos foram superiores em crescimento quando comparadas às linhagens puras provenientes das Filipinas.

O rendimento em filé de um peixe depende do peso corporal, sexo, composição corporal (gordura visceral), características anatômicas (relação cabeça/ corpo), grau de mecanização na filetagem, método de filetagem e destreza do operador (CLEMENTS;
LOVELL, 1994; MACEDO-VIÉGAS; SOUZA, 2004). Para esses pesquisadores, a tilápia do Nilo apresenta rendimentos de filé variando de $25,4 \%$ a $42 \%$ e possui uma carne de ótima qualidade, bom paladar, ausência de microespinhas, recomendada para o consumo fresco, desidratado, salgado ou defumado.

A composição química de um pescado é extremamente variável, depende de vários fatores como da época do ano, do tipo, quantidade e qualidade do alimento consumido, do estágio de maturação sexual, da idade e da parte do corpo analisada (CONTRERAS-GUZMÁN, 1994; CASTAGNOLLI, 1979; ARBELÁEZ-ROJAS; FRACALOSSI; FIM， 2002; OETTERER; SIQUEIRA; GRYSCHEK, 2004).

Poucos são os estudos relacionados às características morfométricas, rendimento e composição de filé de peixes de água doce no Brasil, havendo poucos dados que permitam comparar as espécies, avaliar fatores críticos e visualizar o potencial de industrialização. Esses dados são importantes, pois fornecem subsídios às indústrias de processamento e aos piscicultores que podem estimar sua produção econômica (MACEDO-VIÉGAS; SOUZA, 2004). Este estudo teve como objetivo verificar as características morfométricas, rendimento e composição do filé de três linhagens de tilápia do Nilo produzidas e comercializadas no Norte do Paraná, Brasil.

\section{Material e Métodos}

O experimento foi conduzido na Estação de Piscicultura da Universidade Estadual de Londrina 
(EPUEL), Paraná, Brasil; inicialmente durante a fase de reversão e alevinagem em hapas de tela sombrite ( $3 \times 5 \mathrm{~m}$ ) e posteriormente durante a fase de engorda em tanques-rede de arame galvanizado revestido em $\operatorname{PVC}\left(01 \mathrm{~m}^{3}\right)$ alocados aleatoriamente em viveiro de terra de $750 \mathrm{~m}^{2}$.

Foram consideradas como linhagem tailandesa chitralada (Tai) larvas provenientes da segunda geração de um lote importado da Tailândia de uma estação de piscicultura do norte do Paraná e linhagem Local e híbrida (Hbr, macho tailandêsa x fêmea local) larvas provenientes de duas outras estações de piscicultura do norte e oeste do Paraná, respectivamente.

Inicialmente as larvas foram estocadas em três hapas e submetidas ao tratamento hormonal para reversão sexual com uma dieta contendo $60 \mathrm{mg} / \mathrm{kg}$ de 17 alfa-metiltestoterona durante 28 dias. Posteriormente, na fase de alevinagem foram utilizados nove hapas instalados aleatoriamente no viveiro e 100 alevinos de cada linhagem foram distribuídos ao acaso em cada unidade experimental (três repetições por linhagem) e esta fase teve a duração de dois meses. Na engorda a população de peixes em cada tanque-rede foi reduzida para 20 exemplares e os animais transferidos para nove tanques-rede (três repetições por linhagem) por um período de seis meses. Durante o período experimental foram repostas somente as perdas de água por evaporação e infiltração do viveiro de terra. O oxigênio dissolvido e a temperatura da água foram avaliados através de um oxímetro YSI 55; o pH através de um pH eletrônico modelo CE-F 1002; a condutividade elétrica através de condutivímetro digital modelo Analion C 702, a alcalinidade total por titulação ácida usando alaranjado de metila como indicador; o nitrito e nitrogênio amoniacal por espectrofotometria e ortofosfato pelo método espectrofotométrico descrito por Carmouze (1994) e a transparência através de disco de Secchi. Diariamente foram avaliados temperatura e oxigênio e mensalmente os outros parâmetros.
Durante a reversão e alevinagem os peixes foram alimentados com a ração comercial farelada (42\% de proteína bruta, tabela 01) e na recria e engorda com uma ração comercial extruzada de $03 \mathrm{~mm}$ e 06 $\mathrm{mm}$ respectivamente $(30 \%$ de proteína bruta, tabela 01) duas vezes ao dia, às 09:00 e 17:00 horas, de acordo tabela descrita por Wilson (1991).

Tabela 1. Composição aproximada das dietas comerciais utilizadas.

\begin{tabular}{ccc}
\hline Ítem & $\mathbf{4 2} \%$ de PB & $\mathbf{3 0 \%}$ de PB \\
\hline Proteina bruta (min. \%) & 42,0 & 30,0 \\
Umidade (máx. \%) & 12,0 & 12,0 \\
Fibra bruta (máx. \%) & 3,0 & 3,5 \\
Matéria Mineral (máx. \%) & 15,0 & 9,7 \\
Extrato Etéreo (min. \%) & 4,0 & 4,0 \\
Cálcio (máx. \%) & 3,5 & 2,5 \\
Fósforo (min. \%) & 1,2 & 1,2 \\
\hline
\end{tabular}

Suplemento vitamínico mineral (níveis de garantia por quilograma do produto): Vit A: $3.000 \mathrm{UI}$, Vit $\mathrm{D}_{3}: 1.500 \mathrm{UI}$, Vit E: 120 mg, Vit K: 6 mg, Tiamina: 18 mg, Riboflavina: 24 mg, Piridoxina: $18 \mathrm{mg}$, Ácido pantotênico: $72 \mathrm{mg}$, Ácido nicotínico: $108 \mathrm{mg}$, Biotina: 0,2 mg, Ácido Fólico: $3 \mathrm{mg}$, Vitamina $B_{12}: 0,015 \mathrm{mg}$, Vitamina C: $450 \mathrm{mg}$, Colina: 1.200 $\mathrm{mg}$, Inositol: $150 \mathrm{mg}$, Zinco: $80 \mathrm{mg}$, Ferro: $60 \mathrm{mg}$, Manganês: $40 \mathrm{mg}$, Iodo: $4 \mathrm{mg}$, Cobre: $8 \mathrm{mg}$, Cobalto: 0,8 mg, Cromo: 0,6 mg, Selênio: 0,17 mg.

O desempenho produtivo das três linhagens foi determinado por intermédio dos seguintes parâmetros: média de peso inicial, a taxa média de sobrevivência no cultivo, média de peso final, média de ganho em peso total no período de alevinagem e engorda, média de biomassa final de acordo Dan e Little (2000) e Boscolo et al. (2001).

As medidas morfométricas foram obtidas de cada linhagem na última biometria com auxílio de um paquímetro. Estes valores foram transformados em razões ou proporções, sendo as medidas de comprimento do tronco, altura do corpo e comprimento da cabeça proporcional ao comprimento padrão e a medida de altura de cabeça proporcional ao comprimento da cabeça, da seguinte forma: a) comprimento do tronco/comprimento padrão (C.Tr./ 
C.P.); b) altura do corpo/ comprimento padrão (Al.Cor./C.P.); c) comprimento da cabeça/ comprimento padrão (C.Cab./C.P.) e d) altura da cabeça/comprimento da cabeça (Al. Cab./ C. Cab) de acordo Lundstedt, Leonhardt e Dias (1997) e Boscolo et al. (2001).

A retirada do filé foi realizada utilizando um método descrito por Souza (2002), onde o peixe é decapitado e eviscerado, e removem-se as nadadeiras dorsal e anal, faz-se a retirada da pele com alicate e realizase a filetagem (filé sem pele). O filé é obtido a partir da musculatura dorsal, nas duas laterais do peixe no sentido longitudinal, ao longo de toda a extensão da coluna vertebral e costelas. A média da produção de filé para cada linhagem foi determinada pela diferença entre o peso total dos peixes e o peso de filés obtidos.

De cada filé foi retirada uma amostra para a análise de composição bromatológica. Os fragmentos de filé de cada linhagem foram moídos e homogeneizados para a determinação da umidade, em estufa a $105^{\circ} \mathrm{C}$, até peso constante, proteína bruta (método de Kjeldahl), lipídeos (método de Soxleth), cinzas em mufla a $550^{\circ} \mathrm{C}$, até peso constante. Todas estas determinações foram feitas segundo a Adur Outdoor Activities Centre (1990) e os valores obtidos representam a média de três determinações por amostra.

Em todo o experimento a análise de variância (ANOVA) foi complementada pelo teste de Student Newman Keuls (SNK).

\section{Resultados e Discussão}

Os valores médios dos parâmetros físico-químicos da água apresentados na tabela 02 encontram-se dentro do recomendado para o cultivo da tilápia do Nilo, permitindo bom desempenho dos peixes de acordo Huet (1983), Brune e Tomasso (1991), Boyd (1995) e Kubitza (2000).

Tabela 2.Valores médios dos parâmetros físico-químicos da água do viveiro.

\begin{tabular}{lc}
\hline Variáveis & Média \pm SE \\
\hline Temperatura da água $\left({ }^{\circ} \mathrm{C}\right)$ & $24,51 \pm 4,62$ \\
$\mathrm{pH}$ & $7,54 \pm 0,43$ \\
Alcalinidade $\left(\mathrm{mgCaCO}_{3} / \mathrm{L}\right)$ & $39,64 \pm 9,72$ \\
Condutividade $(\mu \mathrm{mhos} / \mathrm{cm})$ & $70,76 \pm 19,71$ \\
Oxigênio dissolvido $(\mathrm{mg} / \mathrm{L})$ & $7,72 \pm 1,34$ \\
Amônia total $(\mathrm{mg} / \mathrm{L})$ & $0,09 \pm 0,08$ \\
Amônia não ionizada(mg/ $(\mathrm{L})$ & $0,003 \pm 0,003$ \\
Nitrito $(\mathrm{mg} / \mathrm{L})$ & $0,10 \pm 0,01$ \\
Fósforo total dissolvido $(\mathrm{mg} / \mathrm{L})$ & $0,04 \pm 0,01$ \\
Transparência pelo disco de Secchi $(\mathrm{cm})$ & $77,10 \pm 14,52$ \\
\hline
\end{tabular}

Os valores médios de desempenho dos peixes das três linhagens estão apresentados na tabela 03. Os maiores valores para peso final, ganho em peso e biomassa final $(P<0,05)$ foram encontrados para a linhagem Tai, seguida da Local e Hbr, respectivamente. 
Tabela 3. Peso inicial (g), peso final (g), ganho em peso (g), biomassa final $\left(\mathrm{kg} / \mathrm{m}^{3}\right)$ e sobrevivência (\%) das três linhagens de tilápia do Nilo.

\begin{tabular}{lccc}
\hline & Tai & Local & Hbr \\
\hline Peso inicial $(\mathrm{g})$ & $0,39 \pm 0,20 \mathrm{a}$ & $0,41 \pm 0,22 \mathrm{a}$ & $0,45 \pm 0,15 \mathrm{a}$ \\
Peso Final $(\mathrm{g})$ & $650,67 \pm 125,58 \mathrm{a}$ & $534,25 \pm 92,09 \mathrm{~b}$ & $360,00 \pm 62,77 \mathrm{c}$ \\
Ganho em peso $(\mathrm{g})$ & $650,21 \pm 70,08 \mathrm{a}$ & $533,86 \pm 24,70 \mathrm{~b}$ & $360,25 \pm 33,09 \mathrm{c}$ \\
Biomassa final $\left(\mathrm{kg} / \mathrm{m}^{3}\right)$ & $12,95 \pm 1,40 \mathrm{a}$ & $10,68 \pm 0,49 \mathrm{~b}$ & $7,21 \pm 0,66 \mathrm{c}$ \\
Sobrevivência $(\%)$ & $86,54 \pm 1,58 \mathrm{a}$ & $87,26 \pm 0,46 \mathrm{a}$ & $84,67 \pm 2,97 \mathrm{a}$ \\
\hline
\end{tabular}

Médias na mesma linha seguidas de letras distintas são significativamente diferentes $(\mathrm{P}<0,05)$ pelo ANOVA.

Souza et al. (2000) compararam a performance da tilápia do Nilo variedade tailandesa em relação a tilápia vermelha israelense e encontraram para a tailandesa maior ganho em peso quando comparada à israelense. Os resultados de desempenho do presente experimento concordam com os observados por Boscolo et al. (2001), os quais demonstraram haver diferença no crescimento das linhagens tailandesa e a comum.

Não foi observada diferença significativa $(P>$ $0,05)$ para a taxa de sobrevivência média no presente experimento. Resultados similares foram obtidos por
Boscolo et al. (2001) e concordam com os dados de Dan e Little (2000), que avaliando linhagens tailandesa, filipina e vitienamita, não observaram diferença na taxa de sobrevivência entre as diferentes linhagens.

Os valores médios das razões morfométricas como comprimento do tronco/comprimento padrão (C.Tr./C.P.), altura do corpo/ comprimento padrão (Al.Cor./C.P.), comprimento da cabeça/ comprimento padrão (C.Cab./C.P.) e altura da cabeça/ comprimento da cabeça (Al. Cab./ C. Cab), estão relacionados na tabela 04 .

Tabela 4. Valores médios das razões morfométricas das três linhagens de tilápia do Nilo.

\begin{tabular}{lcccc}
\hline & Tai & Local & Hbr & CV (\%) \\
\hline C.tr./ Ls. & $0,68 \pm 0,01 \mathrm{a}$ & $0,68 \pm 0,01 \mathrm{a}$ & $0,68 \pm 0,04 \mathrm{a}$ & 4,6 \\
Al.Cor./ Ls. & $0,41 \pm 0,02 \mathrm{a}$ & $0,42 \pm 0,02 \mathrm{a}$ & $0,41 \pm 0,05 \mathrm{a}$ & 7,6 \\
C.Cab./ Ls. & $0,32 \pm 0,01 \mathrm{a}$ & $0,32 \pm 0,01 \mathrm{a}$ & $0,32 \pm 0,04 \mathrm{a}$ & 3,1 \\
Al. Cab./ C.Cab. & $0,97 \pm 0,06 \mathrm{a}$ & $0,91 \pm 0,05 \mathrm{~b}$ & $0,89 \pm 0,05 \mathrm{~b}$ & 3,4 \\
\hline
\end{tabular}

Médias na mesma linha seguidas de letras distintas são significativamente diferentes $(\mathrm{P}<0,05)$ pelo ANOVA.

A análise das razões morfométricas demonstrou não haver diferenças $(\mathrm{p}>0,05)$ para o comprimento do tronco/comprimento padrão; altura do corpo/ comprimento padrão e comprimento da cabeça/ comprimento padrão. No entanto, as médias para a razão altura da cabeça/comprimento da cabeça apresentam diferenças significativas, de tal forma que a linhagem Tai apresentou maior proporção. Uma maior altura de cabeça provavelmente implica em maior peso da cabeça e um menor rendimento de filé, parâmetro importante para a indústria de filetamento de pescado. Diferenças morfométricas em diferentes grupos de tilápia do Nilo foram também observadas por Lundstedt, Leonhardt e Dias (1997).

Boscolo et al. (2001) observaram que os animais de origem tailandesa apresentaram uma maior relação comprimento da cabeça/comprimento padrão e maior relação comprimento da cabeça/altura da cabeça quando comparados à linhagem comum, mostrando que aqueles animais apresentaram uma cabeça maior em relação à comum.

Os valores médios do rendimento de filé e da análise bromatológica das três linhagens estão apresentados na tabela 05 . 
Tabela 5. Valores médios do rendimento em filé e da análise bromatológica das três linhagens de tilápia do Nilo.

\begin{tabular}{lcccc}
\hline & Tai & Local & Hbr & CV (\%) \\
\hline Rendimento em filé (\%) & $36,51 \pm 3,65 \mathrm{~b}$ & $38,00 \pm 3,80 \mathrm{~b}$ & $39,05 \pm 3,90 \mathrm{a}$ & 10,0 \\
Proteína bruta no filé (\%) & $18,48 \pm 0,73 \mathrm{a}$ & $19,33 \pm 0,82 \mathrm{a}$ & $18,47 \pm 0,27 \mathrm{a}$ & 3,5 \\
Lipídeos no filé (\%) & $2,96 \pm 0,23 \mathrm{a}$ & $1,88 \pm 0,33 \mathrm{c}$ & $2,44 \pm 0,22 \mathrm{~b}$ & 10,9 \\
Cinzas no filé (\%) & $1,41 \pm 0,08 \mathrm{a}$ & $1,41 \pm 0,07 \mathrm{a}$ & $1,33 \pm 0,04 \mathrm{a}$ & 2,3 \\
Umidade no filé (\%) & $76,88 \pm 1,92 \mathrm{a}$ & $78,16 \pm 0,86 \mathrm{a}$ & $75,94 \pm 0,88 \mathrm{a}$ & 1,7 \\
\hline
\end{tabular}

Médias na mesma linha seguidas de letras distintas são significativamente diferentes $(\mathrm{P}<0,05)$ pelo ANOVA.

A linhagem Hbr apresentou maior rendimento em filé $(39,05 \%)(P<0,05)$ quando comparada à Local $(38,00 \%)$ e Tai (36,51\%), que não diferiram entre si $(P>0,05)$. Macedo-Viegas, Souza e Kronka (1997) encontraram rendimentos de filé ao redor de $40 \%$ em tilápia do Nilo com peso de 250 a 300 g. Em geral, o rendimento de filé de tilápias acima de $300 \mathrm{~g}$ varia de $32 \%$ a 38\% (MACEDO-VIEGAS; SOUZA; KRONKA, 1997; SOUZA; MARANHÃO, 2001). Peixes menores acumulam menor quantidade de gordura visceral e, portanto, maior rendimento de filé, pois os cálculos são realizados em função do peixe inteiro (MACEDO-VIÉGAS; SOUZA, 2004).

Souza e Maranhão (2001) observaram que não houve diferença significativa para o rendimento em filé de duas categorias de peso de tilápia do Nilo, $O$. niloticus. Sendo que a primeira categoria situava-se entre 300 e $400 \mathrm{~g}$ e a segunda categoria entre 401 e $500 \mathrm{~g}$, onde foram encontrados respectivamente valores para rendimento em filé de $36,50 \%$ e $36,84 \%$.

Souza, Macedo-Viégas e Kronka (1999) avaliando o rendimento em filé e dois métodos de filetagem manual concluíram que o método de retirar a pele com o alicate e depois o filé proporcionou maior rendimento (36,36\%), quando comparado ao método de filetar e depois retirar a pele $(32,89 \%)$ e que duas faixas de peixes menores (250 a 300 e 301 a $350 \mathrm{~g}$ ) apresentaram os melhores rendimentos de filé. Este método de filetagem e a destreza do filetador provavelmente contribuíram com as altas taxas de rendimento de filé observadas no presente experimento $(36,51 \%$ a $39,05 \%)$.
Boscolo et al. (2001) observaram que as tilápias tailandesas apresentaram rendimento em filé similar $(P>0,05)$ a linhagem comum. Estes resultados estão de acordo aos obtidos no presente experimento. Maior rendimento de filé para a linhagem Hbr no presente experimento pode ser explicado pelo menor peso dos peixes, o que está de acordo com Macedo-Viegas, Souza e Kronka (1997), Souza, Macedo-Viégas e Kronka (1999) e Macedo-Viégas e Souza (2004). Entretanto, Ribeiro et al. (1998) encontraram maior rendimento em filé para tilápias maiores.

Rutten, Bovenhuis e Komem (2004) pesquisaram o rendimento em filé de três linhagens de Oreochromis niloticus tailandesas; a linhagem Chitralada, IDRC e a GIFT. Os peixes foram cultivados em sistema superintensivo $\left(200 \mathrm{~kg} / \mathrm{m}^{3}\right)$ de recirculação de água e alimentados "ad libitum" e ao final do cultivo apresentavam peso final próximo de 700 g. A linhagem chitralada apresentou um rendimento em filé de $34,5 \%$, a IDRC $35,2 \%$ e a GIFT 37,8\% com diferenças significativas $(\mathrm{P}<0,05)$ entre as três linhagens.

A linhagem Tai no presente experimento apresentou menor rendimento em filé, o que está de acordo com o obtido por Rutten, Bovenhuis e Komem (2004). Uma hipótese que pode ser levantada é que esta linhagem apresenta maior tamanho de cabeça, o que também foi confirmado por Boscolo et al. (2001), pois estes pesquisadores demonstraram que a linhagem comum apresentou um rendimento de tronco de $51,39 \%$ e a tailandesa de 49,46\% $(\mathrm{P}<0,05)$. Para Boscolo et al. (2001) as tilápias comuns apresentaram-se com troncos 
mais robustos, pois as razões largura do corpo/ comprimento do tronco e largura do corpo/altura do corpo apresentaram valores superiores $(\mathrm{P}<0,05)$ quando comparadas às tailandesas.

Os valores de rendimento de filé encontrados no presente experimento (36,51\% a 39,05\%) são maiores que os obtidos por Clements e Lovell (1994), Ribeiro et al. (1998), Rutten, Bovenhuis e Komem (2004) e estão próximos aos obtidos por Souza e Maranhão (2001).

O conhecimento da composição centesimal do filé da tilápia é necessário para que o seu consumo possa ser estimulado, possibilitando a competição com outras fontes protéicas largamente utilizadas como a de carne bovina, suína e de aves. A composição centesimal de filés de duas espécies de tilápia foi apresentada por Oetterer, Siqueira e Gryschek (2004), mostrando para tilápia vermelha valores de $16,62 \%$; $1,68 \% ; 1,07 \%$ e $79,20 \%$ enquanto para tilápia do Nilo valores de $17,08 \% ; 1,99 \% ; 1,09 \%$ e $78,43 \%$ respectivamente para proteína bruta, lipídeos, cinzas e umidade, com diferenças $(\mathrm{P}<0,05)$ para proteína bruta, lipídeos e umidade. Os valores obtidos por estes autores e os apresentados por Contreras-Guzmán (1994) estão próximos do obtido no presente estudo. Maiores teores de gordura $(5,7 \%)$ no filé da tilápia do Nilo foram encontrados por Clements e Lovell (1994) em sistemas intensivos circulares de raceways e também por Cho; Jae-Yoon (2002) em sistemas intensivos de recirculação de água.

Menor deposição de gordura nos filés da tilápia do Nilo da linhagem local $(\mathrm{P}<0,05)$ no presente estudo provavelmente torna este produto de maior aceitação no mercado consumidor. De acordo Jacquot (apud CONTRERAS-GUZMÁN, 1994) utiliza-se o teor de gordura como critério prático para comparações entre diferentes pescados. Assim, fala-se em pescado gordo quando o teor de lipídeos mínimo é de $10 \%$, pescado semi-gordo para valores entre 2,5 a $10 \%$ e pescado magro com no máximo de 2,5\% de gordura. Desta forma, o teor de gordura do filé de tilápia do Nilo da linhagem Tai (2,96 \%) faz com que a mesma se torne um produto semi-gordo, enquanto as linhagens Local
$(1,88 \%)$ e $\mathrm{Hbr}(2,44 \%)$ produtos magros. O teor de gordura é de importância na vida útil dos produtos e vital para a aceitação geral pelos consumidores.

\section{Conclusões}

A tilápia do Nilo linhagem tailandesa apresentou maior ganho em peso e também maior tamanho de cabeça quando comparada às linhagens local e híbrida, e ainda apresentou menor rendimento de filé. O teor de gordura no filé da linhagem local é inferior aos dos filés obtidos pela linhagem híbrida e tailandesa. Menor deposição de gordura nos filés da tilápia do Nilo da linhagem local provavelmente tornará este produto de maior aceitação no mercado consumidor.

\section{Referências}

ABUCAY, J. S.; MAIR, G. C.; SKIBINSKI, D. O. F.; BEARDMORE, J. A. Environmental sex determination: the effect of temperature and salinity on sex ratio in Oreochromis niloticus L. Aquaculture, Amsterdam, v.173, p.219-234, 1999.

ADUR OUTDOOR ACTIVITIES CENTRE(AOAC).Official methods of analysis of association of official analytical chemists. 15 ed. Arlington, 1990.

ARBELÁEZ-ROJAS, G. A.; FRACALOSSI, D. M.; FIM, J. D. I. Composição corporal do tambaqui, Colossoma macropomum, e Matrinxã, Brycon cephalus, em sistemas de cultivo intensivo, em igarapé, e semi-intensivo, em viveiros. Revista Brasileira de Zootecnia, Viçosa, v.31, n.3, p.1059-1069, 2002.

BENIGA, Z. M.; CIRCA, A. V. Growth performance evaluation of genetically improved Nile tilapia (Oreochromis niloticus $L$.) in floating cages in Lake Sebu, South Cotabo, Philippines. In: TILAPIA AQUACULTURE, 1., 1997, Ithaca, New York. Proceedings... New York: Fitzsimmons, 1997.p.116-128.

BOSCOLO, W.R.; HAYASHI, C.; SOARES, C. M.; FURUYA, W. M.; NAGAE, M. Y. Desempenho e características de carcaça de machos revertidos de tilápia do Nilo, Oreochromis niloticus, linhagens tailandesa e comum, nas fases inicial e de crescimento. Revista Brasileira de Zootecnia, Viçosa, v.30, n.5, p.1391-1396, 2001.

BOYD, C. E. Bottom soils, sediment, and pond aquaculture. New York: Chapman and Hall,1995. 
BRUNE, D. E.; TOMASSO, J. R. Aquaculture and water quality. Baton Rouge (Lousiana): The World Aquaculture Society, 1991.

CARMOUZE, J. P. O metabolismo dos ecossistemas aquáticos: fundamentos teóricos, métodos de estudo e análises químicas. São Paulo: Edgard Blücher, 1994.

CASTAGNOLLI, N. Fatores que influenciam a absorção de energia nos peixes. In: Fundamentos de nutrição de peixes. São Paulo: Livroceres, 1979.

CLEMENTS, S; LOVELL, R.T. Comparison of processing yields and nutrient composition of culture Nile tilapia (Oreochromis niloticus) and channel catfish (Ictalurus punctatus). Aquaculture, Amsterdam, v.119, p.299-310, 1994.

CONTRERAS-GUZMÁN, E. S. Bioquímica de pescados e derivados. Jaboticabal: Funep, 1994.

CHO, S. H.; JAE-YOON. JO. Effects of dietary energy level and number of meals on growth and body composition of Nile tilapia, Oreochromis niloticus L. during summer and winter seasons. Journal of the World Aquaculture Society, Baton Rouge, v.33, p.48-56, 2002.

DAN, N.C.; LITTLE, D.C. The culture performance of monosex and mixed-sex new-season and overwintered fry in three strains of Nile tilapia (Oreochromis niloticus) in northern Vietnam. Aquaculture, Amsterdam, v. 184, p. 221231,2000 .

HUET, M. Tratado de piscicultura. Madrid: Ediciones Mundi-Prensa, 1983.

KUBITZA, F. Tilápia: tecnologia e planejamento na produção comercial. Jundiaí: Fernando Kubitza, 2000.

LUNDSTEDT, L.M.; LEONHARDT, J. H.; DIAS, A. L. Alterações morfométricas induzidas pela reversão sexual em tilápia do Nilo, Oreochromis niloticus (Linnaeus, 1757). Revista Unimar, Maringá, v.19, n.2, p.461-472, 1997

MACEDO-VIÉGAS, E. M.; SOUZA, M. L. R.; KRONKA, S. N. Estudo da carcaça de tilápia do Nilo, Oreochromis niloticus, em quatro categorias de peso. Revista Unimar, Maringá, v. 19, p. 863-870, 1997.

MACEDO-VIÉGAS, E. M.; SOUZA, M. L. R. Préprocessamento e conservação do pescado produzido em piscicultura. In: CYRINO, J. E. P.; URBINATI, E. C.; FRACALOSSI, D. M.; CASTAGNOLLI, N. (Ed.) Tópicos Especiais em Piscicultura de Água Doce Tropical Intensiva. São Paulo: TecArt, 2004. Cap.14, p.405-480,

MAIR, G. C.; ABUCAY, J. S.; BEARMORE J. A.; SKIBINSKI D. O. F Growth performance trials of genetically male tilapia (GMT) derived from YY-males in Oreochromis niloticus L.: On station comparisons with mixed sex and sex reversed male populations. Aquaculture, Amrsterdam, v.137, p.313-322, 1995.

OETTERER, M.; SIQUEIRA, A A. Z. C.; GRYSCHEK, S. B. Tecnologias emergentes para processamento do pescado produzido em piscicultura. In: CYRINO, J.E. P.; URBINATI,
E. C.; FRACALOSSI, D. M.; CASTAGNOLLI, N. (Ed.). Tópicos Especiais em Piscicultura de Água Doce Tropical Intensiva. São Paulo: TecArt, 2004. Cap. 15, p. 481-500.

POPMA, T. J.; GREEN, B. W. Sex reversal of tilapia in earthen ponds. Auburn (Alabama): Auburn University, Internacional Center for Aquaculture and Aquatic Environments , 1990. (Research And Development, n.35)

POPMA, T. J.; LOVSHIN, L. Wordwide Prospects for Commercial Production of Tilápia. Auburn (Alabama): Auburn University, Internacional Center for Aquaculture and Aquatic Environments.Alabama, 1996. (Research And Development, n.41).

RIBEIRO, L.P.; LIMA, L. C.; TURRA, E. M.; RIBEIRO, T. G. Q.; MIRANDA, M. O. T. Efeito do peso e do operador sobre o rendimento de filé em tilápia vermelha, Oreochromis spp. In: AQUICULTURA BRASIL'98, 2., 1998. Recife. Anais... Recife: SIMBRAQ, 1998. v.2,p.773-778.

RUTTEN, M. J. M.; BOVENHUIS, H.; KOMEN, H. Modeling fillet traits based on body measurements in three Nile tilapia strains (Oreochromis niloticus L.). Aquaculture, Amsterdam, v.231, p.113-122, 2004.

SOUZA, M. L. R.; MACEDO-VIEGAS, E. M.; KRONKA, S. N. Influência do método de filetagem e categorias de peso sobre rendimento de carcaça, filé e pele da tilápia do Nilo, Oreochromis niloticus. Revista Brasileira de Zootecnia, Viçosa, v.28, n.1, p.1-6, 1999.

SOUZA, V. L.; SILVA, P. C.; PÁDUA, D. M. C.; DALACORTE, P. C. Comparison of productive performance of sex reversed male Nile tilapia, Oreochromis niloticus (Thai Strain) and tetra hybrid red tilapia (Israeli Strain). In: INTERNATIONAL SYMPOSIUM ON TILAPIA AQUACULTURE, 5, 2000, Rio de Janeiro. Proceedings... Rio de Janeiro: Ministério da Agricultura e do Abastecimento e Departamento de Pesca e Aqüicultura/Ministério de Agricultura, 2000. v.1, p.83-87.

SOUZA, M. L. R.; MARANHÃO, T. C. F. Rendimento de carcaça, filé e subprodutos da filetagem da tilápia do Nilo, Oreochromis niloticus (L), em função do peso corporal. Acta Scientiarum, Maringá, v.23, n.4, p.897-901, 2001

SOUZA, M. L. R. Comparação de seis métodos de filetagem, em relação ao rendimento de filé e de subprodutos do processamento da tilápia do Nilo (Oreochromis niloticus). Revista Brasileira de Zootecnia, Viçosa, v.31,n.3, p.1076-1084, 2002.

SURESH, A. V. Tilapia update 1998. World Aquaculture, Baton Rouge, v.30, n.4, p.8-13, 1999.

TUAN, P. A.; LITTLE, D. C.; MAIR, G. C. Genotype effect on comparative growth performance of all-male tilapia, Oreochromis niloticus (L.). Aquaculture, Amsterdam, v.159, p.293-302, 1998.

WILSON, R. P. Handbook of nutrient requirements of finfish. Boca Raton: CRC, 1991. 